\title{
Single Frequency Impedance Analysis on Reduced Graphene Oxide Screen-Printed Electrode for Biomolecular Detection
}

\author{
Rajesh $^{1,2}$ (D) Shobhita Singal ${ }^{1,2} \cdot$ Ravinder K. Kotnala $^{1}$
}

Received: 6 January 2017 / Accepted: 9 May 2017 /

Published online: 22 May 2017

(C) Springer Science+Business Media New York 2017

\begin{abstract}
A biofunctionalized reduced graphene oxide (rGO)-modified screen-printed carbon electrode (SPCE) was constructed as an immunosensor for C-reactive protein (CRP) detection, a biomarker released in early stage acute myocardial infarction. A different approach of single frequency analysis (SFA) study was utilized for the biomolecular sensing, by monitoring the response in phase angle changes obtained at an optimized frequency resulting from antigenantibody interactions. A set of measurements were carried out to optimize a frequency where a maximum change in phase angle was observed, and in this case, we found it at around $10 \mathrm{~Hz}$. The bioelectrode was characterized by contact angle measurements, scanning electron microscopy, and electrochemical techniques. A concentration-dependent response of immunosensor to CRP with the change in phase angle, at a fixed frequency of $10 \mathrm{~Hz}$, was found to be in the range of $10 \mathrm{ng} \mathrm{mL}{ }^{-1}$ to $10 \mu \mathrm{g} \mathrm{mL}^{-1}$ in PBS and was fit quantitative well with the HillLangmuir equation. Based on the concentration-response data, the dissociation constant $\left(K_{\mathrm{d}}\right)$ was found to be $3.5 \mathrm{nM}$ (with a Hill coefficient $n=0.57$ ), which indicated a negative cooperativity with high anti-CRP (antibody)-CRP (antigen) binding at the electrode surface. A low-frequency analysis of sensing with an ease of measurement on a disposable electroactive rGO-modified electrode with high selectivity and sensitivity makes it a potential tool for biological sensors.
\end{abstract}

Keywords Frequency Impedance $\cdot$ Phase angle $\cdot$ Protein $\cdot$ Screen-printed electrode $\cdot$ Graphene

Rajesh

rajesh_csir@yahoo.com

1 CSIR-National Physical Laboratory, Dr. K.S. Krishnan Road, New Delhi 110012, India

2 Academy of Scientific and Innovative Research, New Delhi 110012, India 


\section{Introduction}

C-reactive protein (CRP) is a pentameric plasma protein with molecular weight $118 \mathrm{kDa}$, and is considered to be a valuable marker of inflammation indicating incident myocardial infarction, stroke, and sudden cardiac death [1-3]. The American Heart Association has suggested three different CRP concentration levels in human blood serum to evaluate the cardiovascular disease risk: a low-risk state for CRP concentrations less than $1 \mu \mathrm{g} \mathrm{mL}^{-1}$, an average risk state for CRP concentrations between 1 and $3 \mu \mathrm{g} \mathrm{mL}^{-1}$, and high-risk state for any concentration above $3 \mu \mathrm{g} \mathrm{mL}^{-1}[4,5]$. Thus, early quantification of CRP concentration is crucial for accurate and timely therapeutic interventions both before and after the occurrence of AMI in patients. Currently, a number of clinical CRP testing methods are available, such as nephelometric [6], turbidimetric [7], and the enzyme-linked immunosorbent assay (ELISA) kits [8]. However, these methods have many disadvantages of timeconsuming, multi-steps, expensive, and require trained personnel [9]. Other methods of CRP quantification based on surface plasmon resonance (SPR) [10], fluorescence [11], and electrochemistry [12] have been reported during the past few years. Among these, electrochemical assay offers the advantage of low cost and high sensitivity. Recently, a number of CRP assays have been reported by electrochemical impedance spectroscopy (EIS) [13-16]. In EIS, a small amplitude AC signal is applied to the electrode in a wide frequency range and measures the corresponding impedance, which has been extensively used in the past to the sensors for wide applications [17-19]. In an EIS biosensor, any biorecognition event is reflected by a change in impedance parameters, e.g., charge transfer resistance $\left(R_{\mathrm{et}}\right)$, capacitance $\left(C_{\mathrm{dl}}\right)$, and phase angles $(-\Phi)$ that can be used for the detection of biomolecules. The measurement of impedance over a wide frequency range is time consuming, especially if it involves a low-frequency range. Single frequency impedance analysis is an EIS technique where a single frequency is used as an excited signal instead of wide frequency range sweep to reduce the complexity in signal acquisition and its processing, which makes it a suitable, simple and inexpensive analysis technique. Thereby, it has a great ability of a fast response, low cost, and low power analysis with an ease of measurement for both the clinical and on field assays.

Several biosensors have been reported recently on screen-printed carbon electrodes (SPCEs) because of its advantages of simplicity, cost-effectiveness, portability, mass production capabilities and disposable nature [20-22]. However, SPCE biofunctionalization is still a critical factor, which requires further improvement through surface modification of SPCE with other electroactive materials. Recent advances in nanomaterials have offered the possibility to further improve the sensing performance of the biosensors in terms of sensitivity and wide range of biomolecular detection [23-25]. Graphene, a two-dimensional nanomaterial having the properties of high surface area, strong mechanical strength, excellent electrical conductivity, and biocompatibility, is a choice for the fabrication of stable and highly sensitive biosensors for the detection of a various analytes [26-30]. Although, several publications have appeared on graphene for sensor applications, no one has yet reported a single low-frequency impedance analysis on rGO-modified SPCE for biomolecular detection of CRP.

In this work, we report a biofunctionalized rGO-modified SPCE as disposable immunosensor and single frequency impedance analysis technique was applied for the low cost, fast, sensitive, and selective detection of C-reactive protein. The $\mathrm{rGO} / \mathrm{SPCE}$ 
was biofunctionalized with a protein antibody, anti-CRP, via a bilinker, 1-pyrenebutyric acid $N$-hydroxy succinimide ester (PyNHS) utilizing carbodiimide cross-linking reaction to form the anti-CRP-PyNHS/rGO/SPCE. The surface and electrochemical characterization of the immunosensor was done by contact angle measurement, scanning electron microscopy (SEM), cyclic voltammetry, and EIS technique. The analytical characteristic of the immunosensor towards CRP was studied by measuring the phase shift, as an elemental tool, in a single frequency impedance analysis. The changes observed in the phase angle in EIS at a fixed frequency showed a good correlation with CRP concentration and thus provided a high sensitive and selective detection of CRP.

\section{Material and Methods}

\section{Chemicals}

CRP (Cat 8C72), anti-CRP (Cat 4C28), and myoglobin (Cat 8M50) were procured from Hytest (Turku, Finland). PyNHS was procured from Sigma-Aldrich Corp. SPCEs were purchased from $\mathrm{CHI}$ instruments, USA.

\section{Methods}

\section{Preparation of the Bio-SPCE}

The SPCE was first washed with distilled water and dried under $\mathrm{N}_{2}$ gas flow. The SPCE was first modified with graphene oxide (GO) by drop casting a $5-\mu \mathrm{L}$ homogeneous suspension of GO $(0.3 \mathrm{mg} / \mathrm{mL})$ over the working electrode and dried at room temperature for overnight period. After that, it was annealed at $60{ }^{\circ} \mathrm{C}$ for $60 \mathrm{~min}$ to improve the cohesiveness of GO. The GO/SPCE was then electrochemically reduced to $\mathrm{rGO} / \mathrm{SPCE}$ by running 10 successive cyclic voltammetry (CV) cycles within a window of 0 to $-1.4 \mathrm{~V}$ at a scan rate of $50 \mathrm{mV} \mathrm{s}^{-1}$ in $0.5 \mathrm{M} \mathrm{KCl}$ solution purged with $\mathrm{N}_{2}$ gas (Fig. 1). The cathodic current peak at $-1.4 \mathrm{~V}$ is due to the irreversible electrochemical reduction of several oxygen-containing functional groups on the surface of GO [31]. A decrease in the reduction peak with increasing $\mathrm{CV}$ scans to almost a disappearing state confirms a near completion of the reduction of GO to $\mathrm{rGO}$, as evident in Fig. 1. The rGO/SPCE was finally washed with distilled water and dried under $\mathrm{N}_{2}$ gas flow. The $\mathrm{rGO} / \mathrm{SPCE}$ was then incubated with $2 \mu \mathrm{L}$ of $6 \mathrm{mM}$ PyNHS (in DMF) for $1 \mathrm{~h}$ at room temperature, washed with DMF, and dried with $\mathrm{N}_{2}$ gas flow to obtain the PyNHS/rGO/SPCE through $\pi-\pi$ stacking. This was subsequently treated with $5 \mu \mathrm{L}$ PBS containing $1 \mathrm{mg} \mathrm{mL}^{-1}$ anti-CRP at $4{ }^{\circ} \mathrm{C}$ for $2 \mathrm{~h}$, washed with PBS, and dried under $\mathrm{N}_{2}$ gas, to obtain the desired anti$\mathrm{CRP}-\mathrm{Py} / \mathrm{rGO} / \mathrm{SPCE}$ (bio-SPCE). The bio-SPCE was then further treated with $0.1 \%(w / v)$ bovine serum albumin (BSA) to passivate the non-specific binding sites of free esters at bio-SPCE surface and was washed repeatedly with PBS to get rid of any physical adsorption of anti-CRP, dried under $\mathrm{N}_{2}$ gas, and stored at $4{ }^{\circ} \mathrm{C}$. The schematic representation of bio-SPCE fabrication is shown in Fig. 2. 


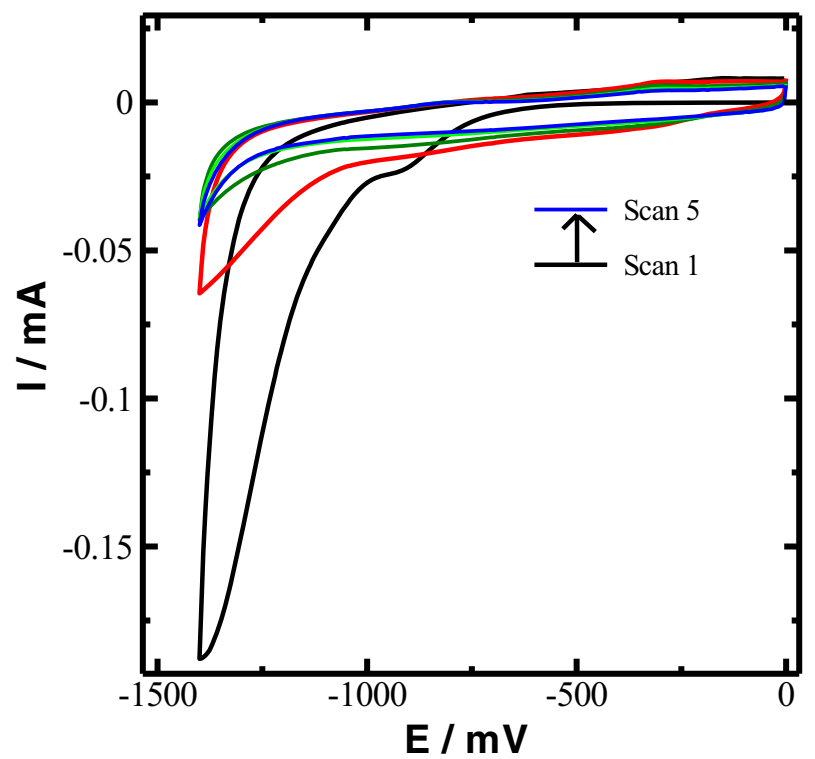

Fig. 1 Electrochemical reduction of GO/SPCE in a deaerated solution of $0.5 \mathrm{M} \mathrm{KCl}$ at a scan rate of $50 \mathrm{mV} \mathrm{s}^{-1}$

\section{Results and Discussion}

\section{Surface Morphology Studies}

Contact angle measurements were carried out to measure the hydrophilic/hydrophobic features of the SPCE surface at each step of modification by static sessile drop method using DSA0MK2 from Kruss GmbH, Germany. Digital snapshots of water droplets on SPCE surface

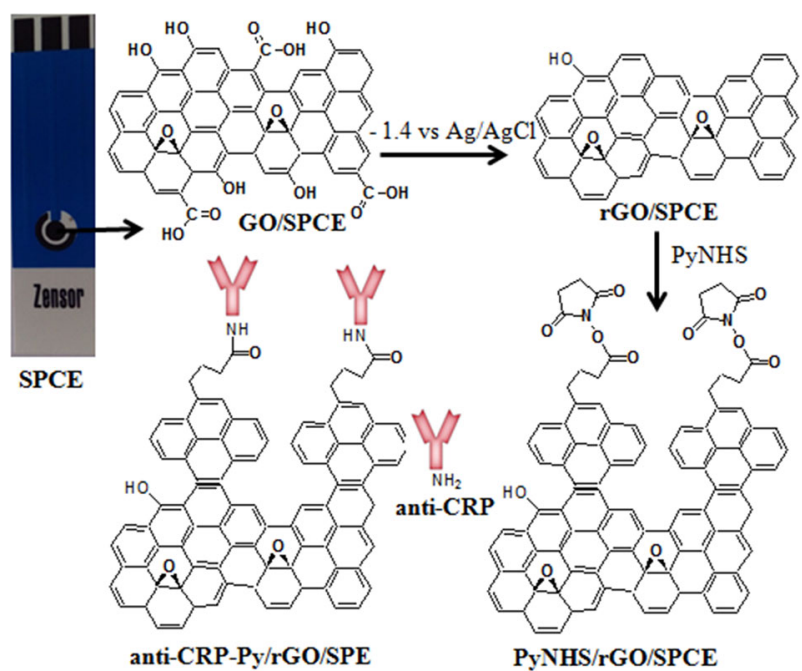

Fig. 2 Schematic representation of the stepwise fabrication of the bioelectrode 

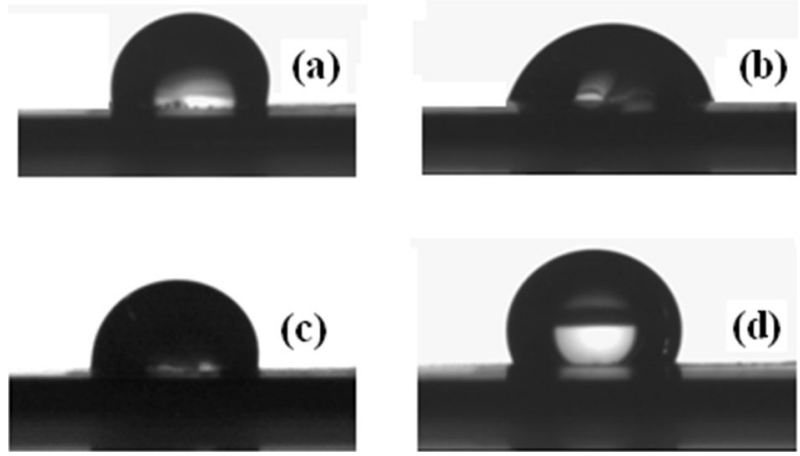

Fig. 3 Contact angle measurement images. a Bare SPCE. b GO/SPCE. c rGO/SPCE. d Anti-CRP-Py/rGO/ SPCE (bio-SPCE)

were taken and analyzed with Kruss software. Three replicate measurements were taken at each modification step to establish the statistical significance. A contact angle of $108.3 \pm 4^{\circ}$ obtained for SPCE surface corresponds to a hydrophobic feature. After the modification of SPCE with GO, the contact angle reduces to $74.2 \pm 3^{\circ}$ (Fig. $3 \mathrm{~b}$ ) due to the presence of the hydrophilic groups like $-\mathrm{OH}$ and $-\mathrm{COOH}$ present at GO surface. Thereafter, an electrochemical reduction of GO/SPCE to $\mathrm{rGO} / \mathrm{SPCE}$ increased the contact angle to $102.4 \pm 3^{\circ}$ (Fig. $3 \mathrm{c}$ ). This was further increased to $118.3 \pm 5^{\circ}$ (Fig. 3d) after the covalent biomolecular immobilization of $\mathrm{rGO} / \mathrm{SPCE}$ with anti-CRP, due to the hydrophobic backbone structure of the protein molecules, which thereby confirmed the fabrication of bio-SPCE.

The surface morphological characterization of the $\mathrm{rGO} / \mathrm{SPCE}$ with and without anti-CRP was done by using SEM images taken from FE-SEM model SUPRA40 VP, Germany. The surface morphologies of the $\mathrm{rGO} / \mathrm{SPCE}$ and anti-CRP functionalized $\mathrm{rGO} / \mathrm{SPCE}$ are shown in Fig. 4. Figure $4 \mathrm{a}$ shows the rGO flakes are uniformly distributed throughout the electrode surface with a porous and wrinkled structure. This highly porous and wrinkled nanostructure of rGO has offered a large active surface area for the biomolecular immobilization of anti-CRP. The appearance of small particulates with a few aggregates over the surface of rGO with a homogeneous feature (Fig. 4b) showed an effective covalent immobilization of anti-CRP indicating to a successful fabrication of bio-SPCE.
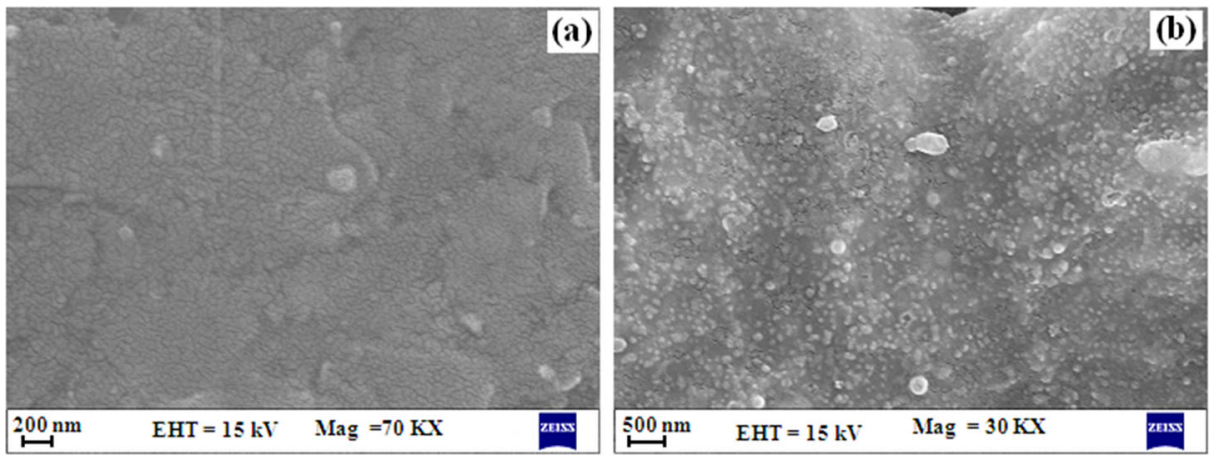

Fig. 4 SEM images. a rGO/SPCE. b anti-CRP-Py/rGO/SPCE 


\section{Electrochemical Characterization of the Bioelectrode Fabrication}

CV studies were carried during each step of surface modification leading to bio-SPCE fabrication using PGSTAT302N, AUTOLAB instrument from Eco Chemie. All CV measurements were performed on SPCE in PBS $(\mathrm{pH} 7.4 ; 0.1 \mathrm{M} \mathrm{KCl})$ containing a mixture of $2 \mathrm{mM}$ $\mathrm{K}_{3}\left[\mathrm{Fe}(\mathrm{CN})_{6}\right]$ and $2 \mathrm{mM} \mathrm{K}_{4}\left[\mathrm{Fe}(\mathrm{CN})_{6}\right]$, as a redox probe, at a scan rate of $50 \mathrm{mV} \mathrm{s}^{-1}$ (Fig. 5a). The bare SPCE showed a reversible CV with a separation between oxidation and reduction potentials $\left(\Delta E_{\mathrm{p}}\right)$ of $241 \mathrm{mV}$. A decrease in the redox peak current with $\Delta E_{\mathrm{p}}=341 \mathrm{mV}$ was observed upon modification of SPCE with GO, showing a sluggish electron transfer characteristic arises due to the two reasons: (i) GO is less electrical conductive because of oxidized surface and (ii) electrostatic repulsion between the negatively charged species of $\left[\mathrm{Fe}(\mathrm{CN})_{6}\right]^{3-/ 4}$ - and oxygenated functional groups of GO. After the electrochemical reduction of GO to rGO, the depletion in oxygen-containing moieties resulted to a redox couple of enhanced peaks current with $\Delta E_{\mathrm{p}}=147 \mathrm{mV}$, thereby confirming the formation of $\mathrm{rGO} / \mathrm{SPCE}$. The electroactive surface area of rGO-modified working electrode of SPCE was determined by using the Randles-Sevcik equation, Eq. 1 [32]:

$$
I_{\mathrm{p}}=\left(2.69 \times 10^{5}\right) n^{3 / 2} A D^{1 / 2} \nu^{1 / 2} \mathrm{C}
$$

where $I_{\mathrm{p}}$ is the redox peak current in ampere, $n$ is the number of electrons participated in redox reaction, $A$ is the electroactive surface area $\left(\mathrm{cm}^{2}\right), D$ is the diffusion coefficient of $\mathrm{K}_{3}\left[\mathrm{Fe}(\mathrm{CN})_{6}\right]$ $\left(\mathrm{cm}^{2} \mathrm{~s}^{-1}\right), C$ is the concentration of redox probe $\left(\mathrm{mol} \mathrm{cm}^{-3}\right)$, and $v$ is the scan rate $\left(\mathrm{V} \mathrm{s}^{-1}\right)$. The $\mathrm{rGO} / \mathrm{SPCE}$ surface exhibited a significant increase in electroactivity due to a twofold increase in the electroactive surface area $\left(0.135 \mathrm{~cm}^{2}\right)$ to its geometric area $\left(0.07 \mathrm{~cm}^{2}\right)$. The modification of $\mathrm{rGO} / \mathrm{SPCE}$ with PyNHS causes a decrease in the redox current with an increased $\Delta E_{\mathrm{p}}$ $(343 \mathrm{mV})$ due to hydrophobic pyrene moiety that perturbed the electron transfer kinetics at the electrode surface. This, upon subsequent immobilization with anti-CRP and passivation of the non-specific sites with BSA, further increased the $\Delta E_{\mathrm{p}}=620 \mathrm{mV}$ with a slowdown in the electron transfer kinetics due to insulating character of protein molecule, indicating the formation of bio-SPCE.
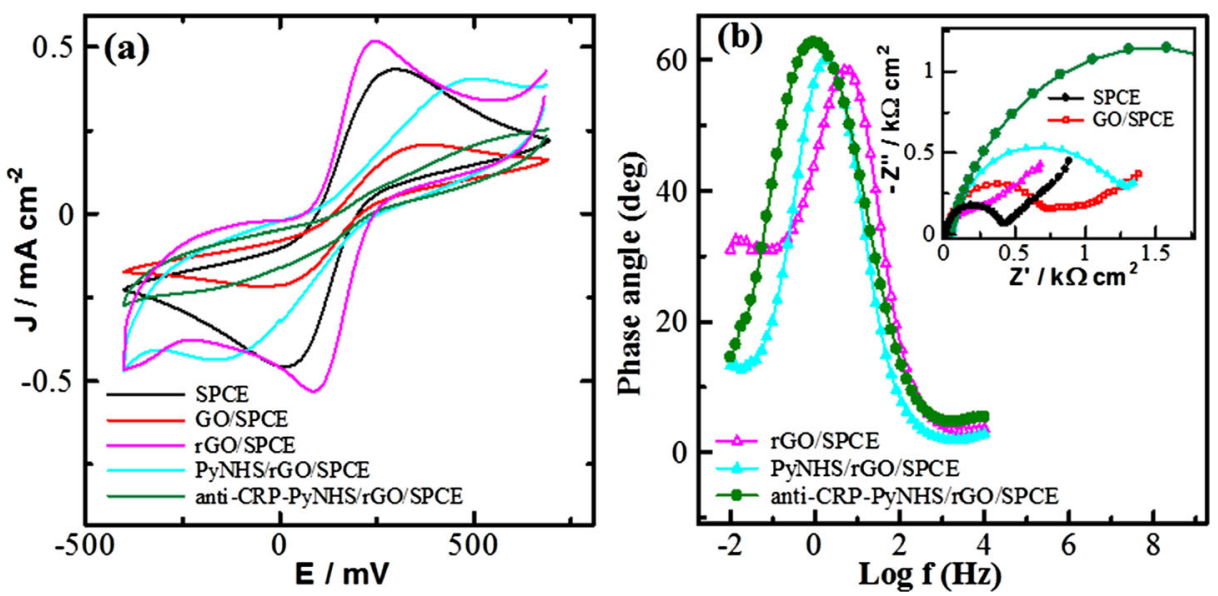

Fig. 5 a $\mathrm{CV}$ of the bioelectrode at different stages of fabrication in $\mathrm{PBS}(0.1 \mathrm{M} \mathrm{KCl}$; $\mathrm{pH} 7.4)$ containing $2 \mathrm{mM}$ $\left[\mathrm{Fe}(\mathrm{CN})_{6}\right]^{3-/ 4-}$; scan rate $50 \mathrm{mV} \mathrm{s}^{-1}$, third $\mathrm{CV}$ scan is shown. b Corresponding Bode plot. Inset: corresponding Nyquist plot 
EIS was also performed to study the interfacial impedance characteristics of the bio-SPCE. According to EIS, a Bode plot is composed of (i) a high-frequency region, (ii) a low-frequency region, and (iii) a mid-frequency region describing the solution properties, the electron transfer characteristics, and the electrode/solution interfacial capacitive properties, respectively. The mid-frequency region is the region where voltage vs time $(V / t)$ and current vs time $(I / t)$ are not in phase and are frequency dependent. Since all the faradic and non-faradic processes occur in this mid frequency region, thus we take it as a region of interest in the present study. The complex impedance possesses both the real (magnitude) and the imaginary (phase) part and can be represented by Eq. (2)

$$
Z^{\prime}=Z e^{i \Phi}
$$

where the real part $Z$ represents the amplitude of voltage to current ratio and $\Phi$ represents the phase difference between the voltage and the current with time, and $i$ is the imaginary unit. Figure $5 \mathrm{~b}$ shows the Bode plot of phase shift with logarithm frequency over the range of $0.01 \mathrm{~Hz}$ to $10 \mathrm{kHz}$ taken during the fabrication of bio-SPCE. The maxima in the phase shift (corresponding to capacitive reactance) of the rGO/SPCE increased and shifted to a lower frequency region after a subsequent functionalization with a bilinker, PyNHS, and anti-CRP. The $\mathrm{rGO} / \mathrm{SPCE}$ showed a maximum phase angle of $58.4^{\circ}$ at $5.2 \mathrm{~Hz}$, which get shifted towards a low-frequency of $0.7 \mathrm{~Hz}$ with an increase in the maximum phase angle to $63.4^{\circ}$ after biomolecular immobilization with anti-CRP, demonstrating the biocompatible feature of the electrode. This is in conformity to a complementary study of charge transfer resistance $\left(R_{\mathrm{et}}\right)$ in Nyquist plot obtained during bio-SPCE fabrication (Inset of Fig. 5b). The chi-squared function $\left(\chi^{2}\right)$ of the best fit equivalent circuit to the Nyquist and Bode plots is the measure of the standard deviation between the original data and the calculated spectrum; this was found to be 1.89 for the bioelectrode, and the detail of other EIS characteristics parameters are given in Table 1.

\section{EIS Response of the Bio-SPCE to Target CRP}

The formation of a complex between the biomolecular probe (antibody) and the complementary antigen molecule upon immunoreaction causes an increase in the phase angle of the circuit. Therefore, a quantitative analysis of CRP can be performed by monitoring the changes in the phase angle upon bio-recognition of different CRP concentrations, at the electrode surface, at an optimized frequency. Frequency analysis study was performed on bio-SPCE, both before and after incubation with target protein antigen, CRP, to find out an optimum

Table 1 CV and EIS characteristics parameters of SPCE at various stages of its surface modification

\begin{tabular}{llllllll}
\hline Type of electrodes & $\begin{array}{l}\Delta E_{\mathrm{p}} \\
(\mathrm{mV})\end{array}$ & $\begin{array}{l}R_{\mathrm{et}} \\
\left(\Omega \mathrm{cm}^{2}\right)\end{array}$ & $\begin{array}{l}\mathrm{CPE} Y_{0} n \\
\left(\mu \mathrm{F} \mathrm{cm} \mathrm{cm}^{-2}\right)\end{array}$ & $\begin{array}{l}k^{0}\left(\mathrm{~m} \mathrm{~s}^{-1}\right) \\
\left(\times 10^{-5}\right)\end{array}$ & $\begin{array}{l}Z_{\mathrm{w}}\left(\Omega \mathrm{cm}^{2}\right) \\
\left(\times 10^{-5}\right)\end{array}$ & $\begin{array}{l}\chi^{2} \\
\left(\times 10^{-1}\right)\end{array}$ & $\begin{array}{l}(-\Phi) \\
\text { Bare SPCE }\end{array}$ \\
GO/SPCE & 241 & 403.2 & 0.120 .93 & 6.72 & 2.33 & 1.21 & 66.6 \\
rGO/SPCE & 341 & 791.7 & 0.070 .88 & 3.42 & 2.23 & 3.10 & 67.6 \\
PyBuNHS/rGO/SPCE & 147 & 286.3 & 13.20 .87 & 9.46 & 2.78 & 4.33 & 57.9 \\
Anti-CRP-PyBu/rGO/SPCE & 620 & 357.4 & 10.70 .91 & 2.78 & 4.85 & 4.11 & 60.1 \\
\hline
\end{tabular}

$\Delta E_{p}$ redox potential, $R_{e t}$ charge transfer resistance, $C P E$ constant phase element, $k^{0}$ apparent rate constant, $Z_{w}$ Warburg impedance, $-\Phi$ highest phase angle 
frequency where a maximum shift in the phase angle was observed. The interaction of target CRP to bio-SPCE was examined in a mixture of $0.1 \mathrm{M} \mathrm{KCl}$ and $2 \mathrm{mM}\left[\mathrm{Fe}(\mathrm{CN})_{6}\right]^{3-/ 4-}$ in PBS $(0.1 \mathrm{M}, \mathrm{pH}$ 7.4). A complete scan of phase angle was performed for immunoreaction in a frequency region of $10 \mathrm{kHz}$ to $0.01 \mathrm{~Hz}$ at bio-SPCE surface. Figure 6a represents the frequency-dependent phase angle Bode plot of the bio-SPCE before and after the bioreaction with $1.0 \mu \mathrm{g} \mathrm{mL}^{-1} \mathrm{CRP}$. Depending on maximum shift observed in the phase angle at $10 \mathrm{~Hz}$, it was chosen as an optimum frequency (inset Fig. 6a), for the concentrationdependent phase angle analysis for CRP detection. Real-time SF impedance analysis was carried out to characterize the binding affinity and the optimization of the bio-reaction time required for the completion of immunoreaction between the bio-receptor and the CRP. For this purpose, a $30 \mu \mathrm{L}$ drop of $1 \mu \mathrm{g} \mathrm{mL}^{-1}$ target CRP analyte in a PB solution of $2 \mathrm{mM}\left[\mathrm{Fe}(\mathrm{CN})_{6}\right]^{3}$ ${ }^{-/ 4-}$ was drop-casted on bio-SPCE and single frequency impedance phase analysis was carried out, at $10 \mathrm{~Hz}$, for $1200 \mathrm{~s}$. The experimental results indicate that more than $90 \%$ saturation in the phase angle was reached within $600 \mathrm{~s}$ (Fig. 6b) and thus a bio-reaction time of $600 \mathrm{~s}$ was selected for CRP immunoassay.

The bio-SPCE response was evaluated from $\Delta(\Phi)=\Phi_{a}-\Phi_{b}$ where $\Phi_{b}$ and $\Phi_{a}$ represent the phase angles of the circuit before and after the incubation of bio-SPCE with varying concentration of CRP, respectively. Figure 7 represents the CRP concentration-dependent change in the phase angle of the circuit with error bars showing standard deviation of the
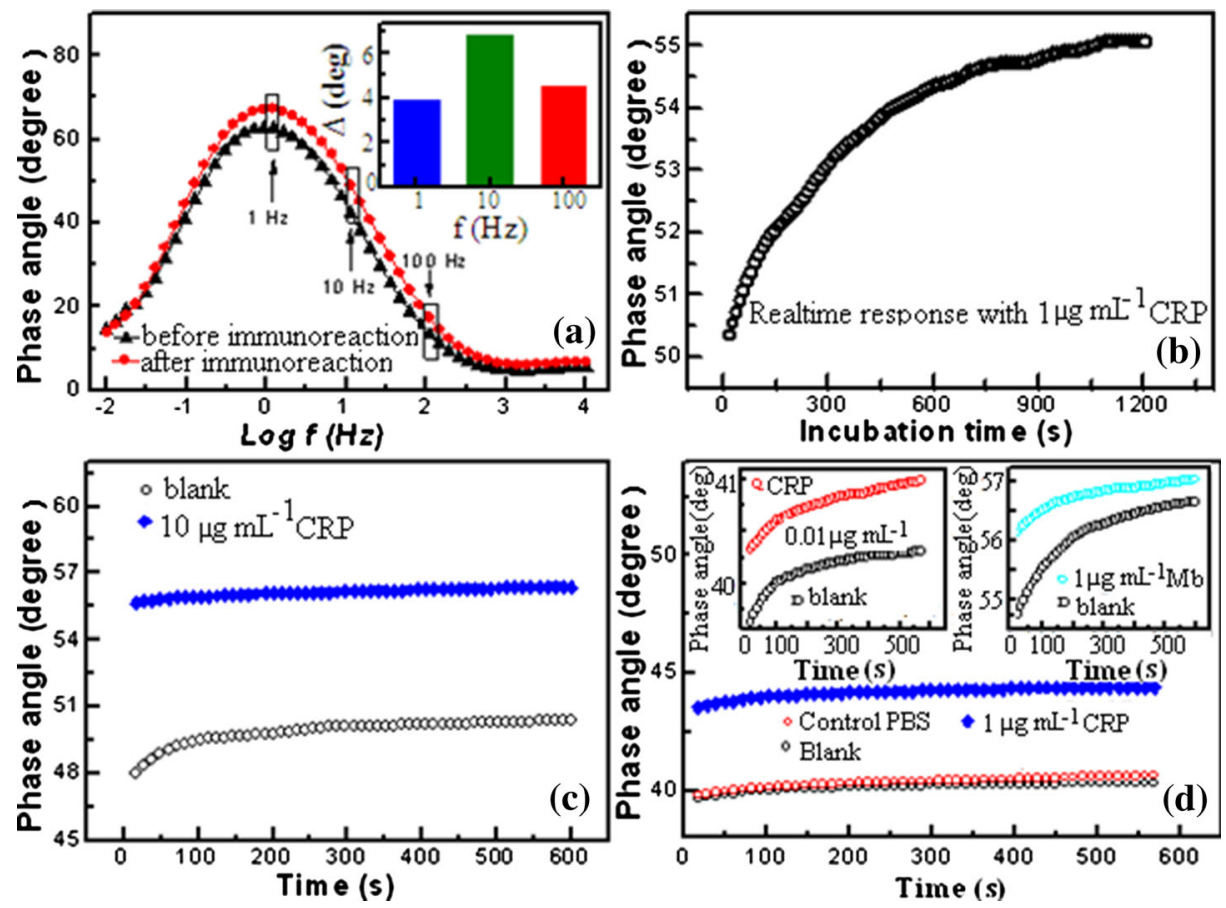

Fig. 6 a Bode plot of the bio-SPCE before and after incubation with $1 \mu \mathrm{g} \mathrm{mL}^{-1} \mathrm{CRP}$ and the inset showing the optimized frequency at which maximum phase shift occur upon interaction. b Single frequency real time response of the bio-SPCE to $1 \mu \mathrm{g} \mathrm{mL}^{-1} \mathrm{CRP}$ at $10 \mathrm{~Hz}$. c SFA of the bio-SPCE for blank (pure PBS) and $10 \mu \mathrm{g} \mathrm{mL}^{-1}$ CRP. d Comparative SFA plot of the bio-SPCE for PBS and $1 \mu \mathrm{g} \mathrm{mL}^{-1} \mathrm{CRP}$; and the inset (upper left) shows a comparative response of bio-SPCE to $0.01 \mu \mathrm{g} \mathrm{mL}^{-1} \mathrm{CRP}$ with PBS and (upper right) to nonspecific $1 \mu \mathrm{g} \mathrm{mL} \mathrm{mb}^{-1} \mathrm{Mith} \mathrm{PBS}$, respectively 


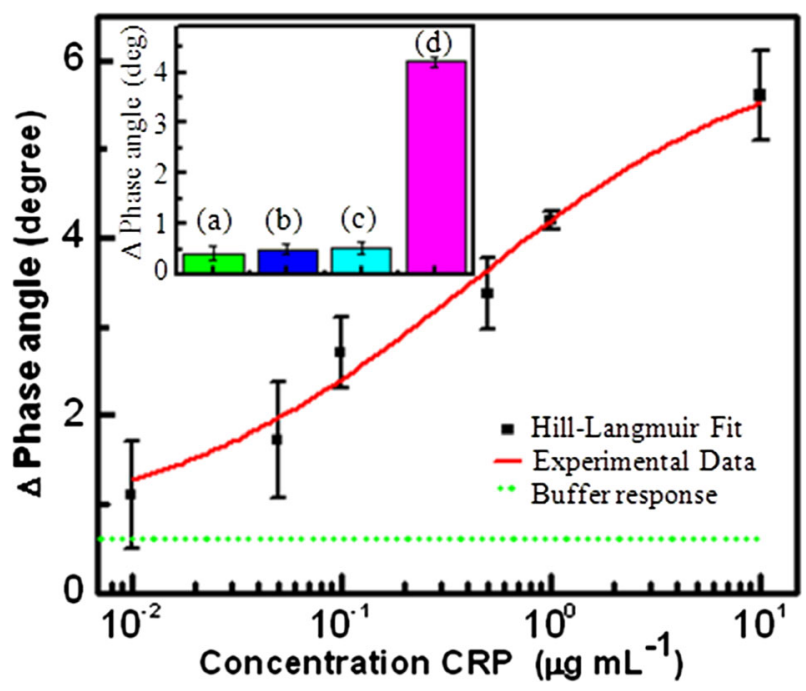

Fig. 7 Calibration curve of bio-SPCE showing changes in phase angle as a function of CRP concentration. The data are fit to a model based on Hill-Langmuir equation (red curve). Inset: Multiple control response of $a$ bioSPCE for PBS buffer; $b$ negative control (PyNHS/rGO/SPCE without anti-CRP) for $1 \mu \mathrm{g} \mathrm{mL}{ }^{-1} \mathrm{CRP}$; $c$ bioSPCE for non-specific $1 \mu \mathrm{g} \mathrm{mL}^{-1} \mathrm{Mb}$; and $d$ bio-SPCE for specific $1 \mu \mathrm{g} \mathrm{mL}^{-1} \mathrm{CRP}$

mean. The concentration-dependent response fitted well with a Hill-Langmuir equation (Eq. 3) model for equilibrium binding between the bio-receptor and the target antigen [33].

$$
\Delta \Phi=A \frac{\left(\frac{c}{K_{d}}\right)^{n}}{1+\left(\frac{c}{K_{d}}\right)^{n}}+Z
$$

where $c, A, Z, K_{\mathrm{d}}$, and $n$ correspond to a CRP concentration, bio-SPCE response having all the binding sites occupied, an offset showing pure buffer response, a dissociation constant revealing a CRP concentration at which half of the binding sites are occupied, and a Hill coefficient accounting the antibody-antigen binding cooperativity, respectively. The curve was fitted to the Hill-Langmuir equation by keeping the maximum response $A$ constrained to a bioSPCE response $(A=5.6)$ and others parameters $Z, K_{\mathrm{d}}$, and $n$ were allowed to vary. It was best fitted with the parameters $K_{\mathrm{d}}=414 \pm 269 \mathrm{ng} \mathrm{mL}^{-1}, Z=0.69 \pm 0.45$, and $n=0.57 \pm 0.07$. The low $K_{\mathrm{d}}$ value of $3.5 \mathrm{nM}$ revealed a strong binding affinity of the target CRP to bio-SPCE. A value of $n=0.57$ indicated a negative cooperativity in CRP binding thereby inhibiting the multiple bonding of CRP antigen to a small aggregate/cluster of anti-CRP on the bio-SPCE surface, possibly due to steric hindrance. The offset parameter $Z=0.69 \pm 0.45$ was in well agreement with the measured bio-SPCE response to the pure buffer $(\Delta \Phi=0.43 \pm 0.15)$, shown by the dotted line in Fig. 7. The response results obtained from a collection of five bioSPCE revealed a clear differentiation between the pure buffer $(\Delta \Phi=0.43 \pm 0.15)$ and the buffer containing $10 \mathrm{ng} \mathrm{mL}^{-1}(84 \mathrm{pM})$ target CRP $\left(\Delta \Phi=1.1 \pm 0.6^{\circ}\right)$, which is about 2.5 times higher for CRP analyte than a pure buffer. This sensitivity of the lowest detection of CRP concentration is sufficient for clinical practice or point of care assay and is better than those reported recently [34, 35]. Similar experiments were carried out with multiple controls with pure PBS; negative control without anti-CRP functionalized SPCE and non-specific antigen $\mathrm{Mb}$ and responses were compared with respect to specific target antigen $\mathrm{CRP}$, under identical 
condition, and the results are shown in the inset of Fig. 7. The response $\left(\Delta \Phi=0.53 \pm 0.11^{\circ}\right)$ obtained for $1 \mu \mathrm{g} \mathrm{mL}^{-1}$ non-specific myoglobin $(\mathrm{cMb})$ is comparable to the pure buffer response $\left(\Delta \Phi=0.43 \pm 0.15^{\circ}\right)$ but is far below the response $\left(\Delta \Phi=4.2 \pm 0.1^{\circ}\right)$ obtained for the same concentration of specific CRP, suggesting a negligible contribution of response from non-specific binding. This was further confirmed with a negative control that showed a response $\left(\Delta \Phi=0.5 \pm 0.1^{\circ}\right)$ nearly comparable to a pure buffer, predicting a good specificity for CRP. The bio-SPCE stability in solution was also investigated with at least six repeated response measurements for a fixed sample concentration of $1.0 \mu \mathrm{g} \mathrm{mL} \mathrm{m}^{-1} \mathrm{CRP}$, which falls within the acceptable range of consistent response demonstrating a good stability.

\section{Conclusion}

We demonstrate an antibody functionalized reduced graphene oxide deposited SPCE, as an impedance immunosensor, for highly sensitive and specific quantification of CRP. The graphene with a large surface area was used as an effective transducer for covalent immobilization of a bioreceptor. The analytical performance of the proposed bioelectrode was then assessed in terms of both sensitivity and specificity using single frequency analysis. The SFA technique utilized in this study is fairly sensitive to electrical change occurring at the bio-SPCE surface and allowed sensitive determination of change in phase angle with time. The bio-SPCE exhibited a concentration-dependent response in the range of $10 \mathrm{ng} \mathrm{mL}^{-1}$ to $10 \mu \mathrm{g} \mathrm{mL}^{-1} \mathrm{CRP}$ in PBS which fall well in agreement to Hill-Langmuir equation of equilibrium thermodynamics. This range of detection is much wider than other disposable biosensors recently reported for CRP $[12,13,35]$ and advantageous over a few others designed with other EIS methods $[14,34,35]$ due to its simple, cost-effective, disposable, and on-site analysis features. These results indicate that the SFA electrochemical analysis of the anti-CRP-PyBu/rGO/SPCE may provide a cost-effective point-of-care diagnosis tool for the sensitive detection of CRP after suitable optimization with real clinical sample.

Acknowledgements Director, CSIR-NPL, New Delhi, is acknowledged for research facilities. S Singal is grateful to CSIR for funding (SRF).

\section{References}

1. May, A., \& Wang, T. J. (2007). Evaluating the role of biomarkers for cardiovascular risk prediction: focus on CRP, BNP and urinary microalbumin. Expert Review of Molecular Diagnostics Expert Review of Molecular Diagnostics, 7, 793-804.

2. Miller, V. M., Redfield, M. M., \& McConnell, J. P. (2007). Use of BNP and CRP as biomarkers in assessing cardiovascular disease: diagnosis versus risk. Current Vascular Pharmacology, 5, 15-25.

3. Mygind, N. D., Harutyunyan, M. J., Mathiasen, A. B., Ripa, A. B., Thune, R. S., Gotze, J. J., Johansen, J. P., Kastrup, J. S., \& Grp, J. (2011). The influence of statin treatment on the inflammatory biomarkers YKL-40 and HsCRP in patients with stable coronary artery disease. Inflammation Research, 60, 281-287.

4. Kushner, I., \& Sehgal, A. R. (2002). Is high-sensitivity C-reactive protein an effective screening test for cardiovascular risk? Archives of Internal Medicine, 162, 867-869.

5. Benzaquen, L. R., Yu, H., \& Rifai, N. (2002). High sensitivity C-reactive protein: an emerging role in cardiovascular risk assessment. Critical Reviews in Clinical Laboratory Sciences, 39, 459-497. 
6. Roberts, W. L., Sedrick, R., Moulton, L., Spencer, A., \& Rifai, N. (2000). Evaluation of four automated high-sensitivity C-reactive protein methods: implications for clinical and epidemiological applications. Clinical Chemistry, 46, 461-468.

7. Roberts, W. L., Moulton, L., Law, T. C., Farrow, G., Cooper-Anderson, M., Savory, J., \& Rifai, N. (2001). Evaluation of nine automated high-sensitivity C-reactive protein methods: implications for clinical and epidemiological applications. Clinical Chemistry, 47, 418-425.

8. Macy, E. M., Hayes, T. E., \& Tracy, R. P. (1997). Variability in the measurement of C-reactive protein in healthy subjects: implications for reference intervals and epidemiological applications. Clinical Chemistry, 43, 52-58.

9. Pearson, T. A., Mensah, G. A., Hong, Y. L., \& Smith, S. C. (2004). CDC/AHA workshop on markers of inflammation and cardiovascular disease application to clinical and public health practice: overview. Circulation, 110, 543-544.

10. Hu, W. P., Hsu, H. Y., Chiou, A., Tsens, K. Y., Lin, H. I., Chang, G. L., \& Chen, S. J. (2006). Immunodetection of pentamer and modified C-reactive protein using surface plasmon resonance biosensing. Biosensors \& Bioelectronics, 21, 1631-1637.

11. Luo, Y., Zhang, B., Chen, M., Jiang, T., Zhou, D., Huang, J., \& Fu, W. (2012). Sensitive and rapid quantification of C-reactive protein using quantum dot-labeled microplate immunoassay. Journal of Translational Medicine, 10, 24.

12. Buch, M., \& Rishpon, J. (2008). An electrochemical immunosensor for C-reactive protein based on multiwalled carbon nanotube-modified electrodes. Electroanalysis, 20, 2592-2594.

13. Fakanya, W. M., \& Tothill, I. E. (2014). Detection of the inflammation biomarker C-reactive protein in serum samples: towards an optimal biosensor formula. Biosensors, 4, 340-357.

14. Gupta, R. K., Periyakaruppan, A., Meyyappan, M., \& Koehne, J. E. (2014). Label-free detection of Creactive protein using a carbon nanofiber based biosensor. Biosensors \& Bioelectronics, 59, 112-119.

15. Songjaroen, T., Feeny, R. M., Mensack, M. M., Laiwattanapaisal, W., \& Henry, C. S. (2016). Label-free detection of C-reactive protein using an electrochemical DNA immunoassay. Sensing and Biosensing Research, 8, 14-19.

16. Vermeeren, V., Grieten, L., Bon, N. V., Bijnens, N., Wenmackers, S., Janssens, S. D., Haenen, K., Wagner, P., \& Michiels, L. (2011). Impedimetric, diamond-based immunosensor for the detection of C-reactive protein. Sensors and Actuators B: Chemical, 157, 130-138.

17. Singal, S., Srivastava, A. K., \& Rajesh. (2017). Label-free impedimetric immunosensor for sensitive detection of fenvalerate in tea. Nano-Micro Letters, 9, 7.

18. Park, J., You, X., Jang, Y., Nam, Y., Kim, M., Min, N., \& Pak, J. (2014). ZnO nanorod matrix based electrochemical immunosensors for sensitivity enhanced detection of Legionella pneumophila. Sensors and Actuators B: Chemical, 200, 173-180.

19. Zhu, Z., Shi, L., Feng, H., \& Zhou, H. (2015). Single domain antibody coated gold nanoparticles as enhancer for clostridium difficile toxin detection by electrochemical impedance immunosensors. Bioelectrochemistry, 101, 153-158.

20. Tangkuaram, T., Ponchio, C., Kangkasomboon, T., Katikawong, P., \& Veerasai, W. (2007). Design and development of a highly stable hydrogen peroxide biosensor on screen printed carbon electrode based on horseradish peroxidase bound with gold nanoparticles in the matrix. Biosensors \& Bioelectronics, 22, 20712078.

21. Rao, V. K., Sharma, M. K., Pandey, P., \& Sekhar, K. (2006). Comparison of different carbon ink based screen-printed electrodes towards amperometric immunosensing. World Journal of Microbiology and Biotechnology, 22, 1135-1143.

22. Renedo, O. D., Lomillo, M. A., \& Martinez, M. J. (2007). Recent developments in the field of screenprinted electrodes and their related applications. Talanta, 73, 202-219.

23. Bhardwaj, S. K., Bhardwaj, N., Mohanta, G. C., Kumar, P., Sharma, A. L., Kim, K. H., \& Deep, A. (2015). Immunosensing of atrazine with antibody-functionalized Cu-MOF conducting thin films. ACS Applied Materials and Interfaces, 7, 26124-26130.

24. Deep, A., Bhardwaj, S. K., Paul, A., Kim, K. H., \& Kumar, P. (2015). Surface assembly of nano-metal organic framework on amine functionalized indium tin oxide substrate for impedimetric sensing of parathion. Biosensors \& Bioelectronics, 65, 226-231.

25. Aragay, G., Pino, F., \& Merkoci, A. (2012). Nanomaterials for sensing and destroying pesticides. Chemical Reviews, 112, 5317-5338.

26. Atar, N., Yola, M. L., \& Eren, T. (2016). Sensitive determination of citrinin based on molecular imprinted electrochemical sensor. Applied Surface Science, 362, 315-322.

27. Yola, M. L., Gupta, V. K., \& Atar, N. (2016). New molecular imprinted voltammetric sensor for determination of ochratoxin a. Materials Science and Engineering: C, 61, 368-375. 
28. Rajesh, G. Z., Vishnubhotla, R., Ducos, P., Serrano, M. D., Ping, J., Robinson, M. K., \& Jhonson, A. T. C. (2016). Genetically engineered antibody functionalized platinum nanoparticles modified CVD-graphene nanohybrid transistor for the detection of breast cancer biomarker, HER3. Advanced Materials Interfaces, 3 , 1600124.

29. Singal, S., Srivastava, A. K., Biradar, A. M., Mulchandani, A., \& Rajesh. (2014). Pt nanoparticles-chemical vapor deposited graphene composite based immunosensor for the detection of human cardiac troponin I. Sensors and Actuators B: Chemical, 205, 363-370.

30. Wang, S., Sun, H., Ang, H. M., \& Tade, M. (2013). Adsorptive remediation of environmental pollutants using novel graphene-based nanomaterials. Journal of Chemical Engineering, 226, 336-347.

31. Jian, J. M., Liu, Y. Y., Zhang, Y. L., Guo, X. S., \& Cai, Q. (2013). Fast and sensitive detection of Pb2+ ion in foods using disposable screen-printed electrode modified by reduced graphene oxide. Sensors, 13, $13063-$ 13075.

32. Yola, M. L., Eren, T., \& Atar, N. (2014). A novel and sensitive electrochemical DNA biosensor based on Fe@Au nanoparticles decorated graphene oxide. Electrochimica Acta, 125, 38-47.

33. Hill, A. (1910). The possible effects of the aggregation of the molecules of haemoglobin on its dissociation curves. Journal of Physiology, 40, 4.

34. Bryan, T., Luo, X., Bueno, P. R., \& Davis, J. J. (2013). An optimized electrochemical biosensor for the label-free detection of C-reactive protein in blood. Biosensors \& Bioelectronics, 39, 94-98.

35. Kumar, D., \& Prasad, B. B. (2012). Multi-walled carbon nanotubes embedded molecularly imprinted polymer-modified screen printed carbon electrode for the quantitative analysis of C-reactive protein. Sensors Actuators B-Chemical, 171, 1141-1150. 